\title{
Integrated tRNA, transcript, and protein profiles in response to steroid hormone signaling
}

\author{
KEVIN VAN BORTLE, ${ }^{1}$ MICHAEL H. NICHOLS, EDWARD RAMOS, ${ }^{2}$ and VICTOR G. CORCES \\ Department of Biology, Emory University, Atlanta, Georgia 30322, USA
}

\begin{abstract}
The accurate and efficient transfer of genetic information into amino acid sequences is carried out through codon-anticodon interactions between mRNA and tRNA, respectively. In this way, tRNAs function at the interface between gene expression and protein synthesis. Whether tRNA levels are dynamically regulated and to what degree tRNA abundance influences the cellular proteome remains largely unexplored. Here we profile tRNA, transcript and protein levels in Drosophila Kc167 cells, a plasmatocyte cell line that, upon treatment with 20-hydroxyecdysone, differentiates into macrophages. We find that high abundance tRNAs associate with codons that are overrepresented in the Kc167 cell proteome, whereas tRNAs that are in low supply associate with codons that are underrepresented. Ecdysone-induced differentiation of Kc167 cells leads to changes in mRNA codon usage in a manner consistent with the developmental progression of the cell. At both early and late time points, ecdysone treatment concomitantly increases the abundance of tRNAThr(CGU), which decodes a differentiation-associated codon that becomes enriched in the macrophage proteome. These results together suggest that tRNA levels may provide a meaningful regulatory mechanism for defining the cellular proteomic landscape.
\end{abstract}

Keywords: codon usage bias; ecdysone; Drosophila; small RNA; proteomics

\section{INTRODUCTION}

By surveying tRNA gene copy numbers across several model organisms, studies have shown that the frequency of codon usage positively correlates with the relative abundance for most tRNA species (Percudani et al. 1997; Kanaya et al. 1999; Qian et al. 2012). Highly expressed genes are often enriched for optimal, high-frequency codons (Duret 2002; Goetz and Fuglsang 2005), and optimization of codon usage in a particular transcript can significantly alter the level of protein expression (Gustafsson et al. 2004), suggesting that cellular concentrations of tRNAs may influence the rate of translation. Although a relationship between tRNA abundance and translational efficiency has been generally accepted (Novoa and Ribas de Pouplana 2012), recent ribosome profiling studies do not capture increased ribosomal pausing at rare codons (Ingolia et al. 2011), leading to a renewed debate over this general assumption. A recent alternative hypothesis argues that if aminoacylated tRNAs are in short supply to translating ribosomes, the concentration for each tRNA isoacceptor would become equal when tRNA abundance is balanced with codon usage (Qian et al. 2012), providing a

\footnotetext{
${ }^{1}$ Present address: Department of Genetics, Stanford University, Stanford, CA 94305, USA

${ }^{2}$ Present address: Genome Rush, Snellville, GA 30039, USA

Corresponding author: vcorces@emory.edu

Article published online ahead of print. Article and publication date are at http://www.rnajournal.org/cgi/doi/10.1261/rna.052126.115.
}

possible explanation for the uniformity in ribosome occupancy while accounting for the importance of tRNA abundance in translation efficiency.

The observation that tRNA levels are finely tuned with codon usage across diverse organisms strongly suggests that tRNA abundance plays an important role in optimizing the translational output of the cell. Taking the next step, it is conceivable that within an organism, different cell types may express a unique codon usage that would require dynamic regulation of the tRNA pool. The increasing demand for one codon, for example, would benefit by increasing the relative abundance of the cognate tRNA. A recent survey of tRNA abundance and codon usage across numerous proliferative and differentiated cell types indeed suggests that tRNA levels are dynamically tuned toward codon usage (Gingold et al. 2014). RNA expression analysis shows that proliferative cancer cell lines and cells overexpressing the transcription factor Myc tend to possess a unique codon bias compared with differentiated cells or those with induced senescence. Accordingly, tRNA abundance in differentiated cells is better matched for differentiated codon bias, and vice versa. However, to what degree tRNA expression is modulated in a

\footnotetext{
(C) 2015 Van Bortle et al. This article is distributed exclusively by the RNA Society for the first 12 months after the full-issue publication date (see http:// rnajournal.cshlp.org/site/misc/terms.xhtml). After 12 months, it is available under a Creative Commons License (Attribution-NonCommercial 4.0 International), as described at http://creativecommons.org/licenses/by-nc/4.0/.
} 
single cell type in response to differentiation, and whether or not changes in tRNA abundance produce differences in translational output, remains unsettled.

Here we integrate profiles for tRNA, transcript, and protein levels, and demonstrate that high abundance tRNAs associate with codons that are overrepresented in the proteome of Drosophila melanogaster, providing evidence in support of the translational efficiency hypothesis. Induction of steroidhormone signaling in Kc167 cells, a plasmatocyte cell line characterized for its robust and temporal-specific response to 20-hydroxyecdysone (ecdysone, 20HE), leads to cellular differentiation into macrophages. This is accompanied by changes in codon usage that mirror the differentiation status of the cell. By profiling the transcriptional, small RNA, and proteomic responses to ecdysone at both early and late time points, we identify changes in proteomic codon representation that mirror earlier changes in transcript and tRNA levels. Specifically, we find that $\mathrm{tRNA}^{\text {Thr(CGU) }}$, which decodes a codon enriched in differentiation genes, increases in response to $20 \mathrm{HE}$ at both early and late time points, correlating with an increase in proteomic codon representation. Ecdysone also increases the levels of three tRNAs that decode differentiation-associated codons, and decreases the level of one tRNA decoding a proliferation-associated codon. These results suggest that $\mathrm{tRNA}$ abundance may represent an important regulatory mechanism for shaping the proteome of a cell, and that variation in tRNA levels between cell types and individuals may underlie proteomic diversity across populations.

\section{RESULTS}

\section{Estimation of tRNA abundance in Drosophila Kc167 cells}

To characterize the relationship between tRNA levels, transcript codon usage, and proteomic output, we carried out an integrated analysis of transcript and protein levels in Drosophila Kc167 cells. For analysis of small RNAs, which include low molecular weight noncoding RNAs such as micro RNAs (miRNA), small nucleolar RNAs (snoRNA), ribosomal RNAs (rRNA), and transfer RNAs (tRNA), we enriched for RNA species $<200$ nucleotides (nt) long (Supplemental Fig. S1) and constructed indexed libraries for multiplex RNA sequencing (RNA-seq). RNA-seq reads were mapped to the release 6 reference sequence of the Drosophila melanogaster genome (Hoskins et al. 2015), and the relative abundance for each tRNA and miRNA was determined by the enrichment of sequencing reads mapping to each annotated region.

With respect to tRNA genes, 44 tRNA types, including the selenocysteine isoacceptor, are currently annotated in D. melanogaster and were considered in our analyses. Comparison of tRNA gene copy number with the abundance of tRNAs in Drosophila Kc167 cells, as determined by small RNA-seq, demonstrates a strong relationship between tRNA levels and the number of gene copies for those currently annotated (Fig. 1A, $P=8.1 \times 10^{-6}$, Spearman's rank correlation). Previous comparisons between codon usage and tRNA levels
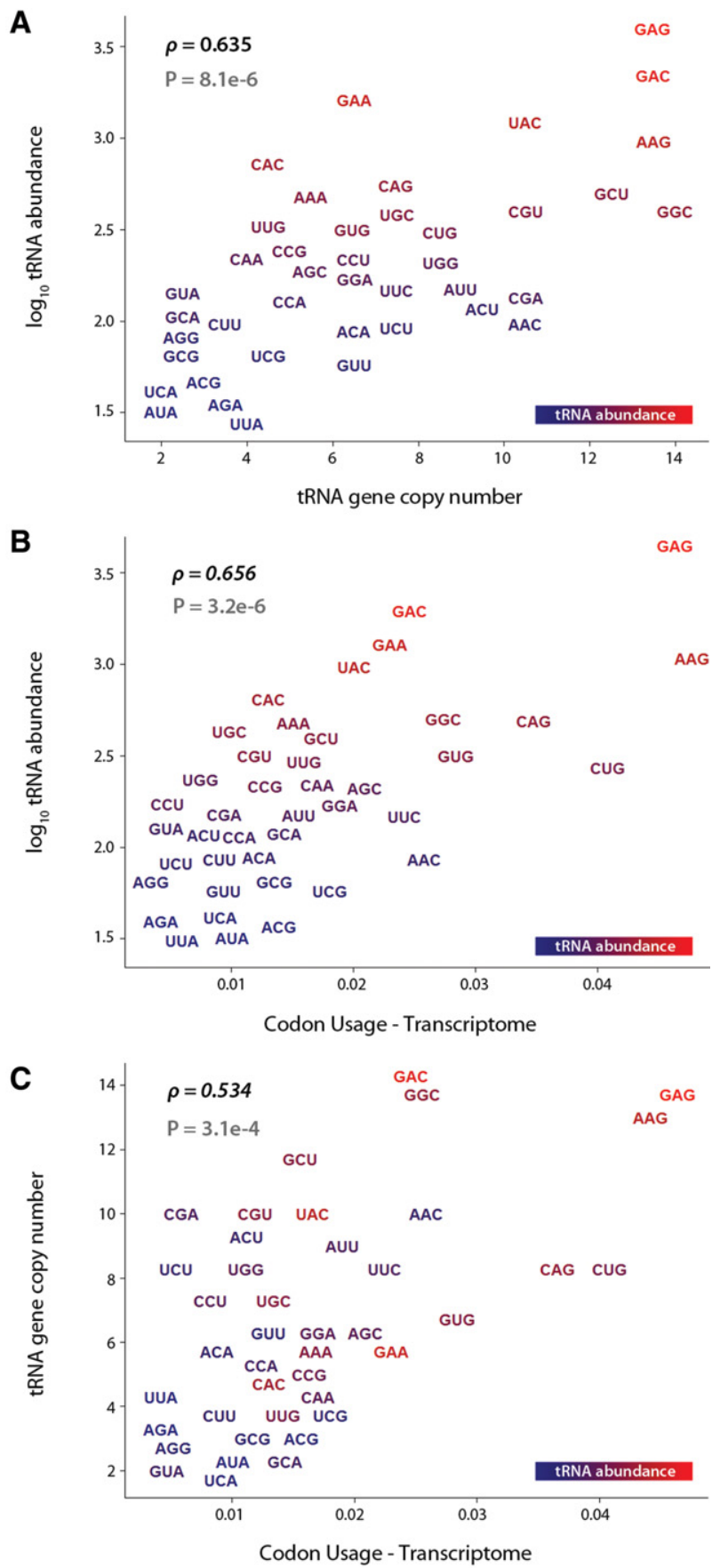

FIGURE 1. Estimation of tRNA abundance in Drosophila Kc167 cells. (A) Correlation plot for tRNA levels ( $\log _{10}$ tRNA abundance, as measured by small RNA-seq experiments; $y$-axis) with tRNA gene copy number ( $x$-axis). $\rho=0.635$; Spearman's rank correlation, $P=8.1 \times$ $10^{-6}$. (B) Correlation plot for tRNA abundance ( $y$-axis) with codon usage weighed by transcript level ( $x$-axis). $\rho=0.656$; Spearman's rank correlation, $P=3.2 \times 10^{-6}$. $(C)$ Analogous correlation plot for tRNA gene copy number ( $y$-axis) with codon usage weighed by transcript level ( $x$-axis). $\rho=0.534$; Spearman's rank correlation, $P=3.1 \times 10^{-4}$. 
relied on organismal tRNA gene copy number as a proxy for tRNA abundance, and these results support this generalized relationship.

We next asked whether tRNA levels are finely tuned toward codon usage, defined as the frequency in which a particular codon is used with respect to all codons. For most organisms, codon usage is determined using the annotated coding sequence within the genome. Though this approach is necessary where the levels of individual transcripts have not been measured, accurate estimation of codon usage must take into consideration the relative number of copies for each messenger RNA (mRNA) within any given cell. Therefore, to accurately quantify codon usage in Drosophila Kc167 cells, we counted the number of occurrences for each specific codon triplet in the transcriptome, and corrected for the relative abundance of each transcript (Supplemental Fig. S2). Comparison of genomic and transcriptomic codon usage reveals that, overall, the representation frequency of each codon is similar in either case (Supplemental Fig. S3). However, differences in codon usage have been discovered in studies comparing proliferative and differentiated tissues (Gingold et al. 2014), and small changes in transcription may have significant effects on codon usage.

We find that the frequency of codon usage specific to Kc167 cells correlates significantly with the abundance of tRNAs measured by RNA-seq (Fig. 1B, $P=3.2 \times 10^{-6}$, Spearman's rank correlation). The frequency of codon usage also shows a significant, albeit weaker, correlation with tRNA gene copy number (Fig. $1 \mathrm{C}, P=3.1 \times 10^{-4}$, Spearman's rank correlation), suggesting that while tRNA gene copy numbers may generally correlate with genomic codon usage in Drosophila, the relative abundance of each tRNA species is more finely tuned to the frequency of codon usage specific to Kc167 cells. Together, these results validate the estimation of tRNA abundance by small RNA-seq and suggest that tRNA levels may be tightly regulated to match the codon representation of a particular cell type. Given the wide range in relative abundance across tRNA molecules, we next asked whether tRNA levels might also influence the proteomic signature of Kc167 cells, potentially by increasing the translational efficiency for codons paired with high abundance tRNA types.

\section{tRNA abundance scales with biased codon representation in the Drosophila proteome}

To compare tRNA abundance with proteomic codon representation, we integrated the small RNA-seq analysis with protein quantification experiments performed on matched cellular lysates (Fig. 2A). We used label-free quantitative mass spectrometry to determine the level of each protein present in Kc167 cells. We then calculated codon representation in the proteome by taking into consideration the number of occurrences for each codon triplet in the translated proteome weighed by the relative abundance for each polypeptide (Supplemental Fig. S2). Comparison of codon usage in the transcriptome with codon representation in the proteome reveals a significant correlation (Fig. $2 \mathrm{~B}, P<2.2 \times 10^{-16}$, Spearman's rank correlation), as one might expect if the proteome is in large part influenced by the relative abundance of mRNAs. However, we find that specific codon triplets are observed in the proteome more or less frequently than expected based simply on transcript codon usage (Fig. 2C). For example, codons AAG (lysine) and GAG (glutamic acid) are counted far more frequently, and codons UGU (cysteine) and AUA (isoleucine) far less frequently in the proteome than their respective codon usage frequencies in the transcriptome. Instead, we find that tRNA abundance is a strong predictor of biased codon representation in the proteomic data (Fig. 2D, $P=6.9 \times 10^{-6}$, Spearman's rank correlation). For example, in agreement with the abundance of the respective codons in the proteome, tRNA ${ }^{\text {Lys(CUU) }}$ and tRNA ${ }^{\text {Glu(CUC) }}$ are two of the most abundant tRNAs in Kc167 cells, whereas tRNA $^{\text {Cys(ACA) }}$ and tRNA ${ }^{\text {Ile(UAU) }}$ are two of the least abundant. More generally, we find that all codons with increased representation in the proteome correlate with tRNAs that are, on average, significantly more abundant, and vice versa (Fig. 2E, $P=5.9 \times 10^{-4}$, Wilcoxon rank sum test). Furthermore, although we do observe a general relationship between transcript codon usage and biased codon representation, we find examples in which high abundance tRNAs increase proteomic codon representation despite low transcript codon usage (e.g., tRNA ${ }^{\operatorname{Arg}(A C G)}$, Fig. 2C,D). The observed relationship between tRNA levels and over- or underrepresentation of cognate codons in the proteome suggests that tRNA abundance may influence the translational flow from transcript to protein.

\section{Transcriptional and proteomic response to steroid- hormone signaling in Kc167 cells}

We next asked whether codon usage and tRNA abundance are significantly altered in response to changes in transcription. In Drosophila, Kc167 cells provide a powerful system for assaying the effects of steroid-hormone signaling, as 20-hydroxyecdysone induces robust morphological changes and transcriptional dynamics that are broken into temporal-specific early and late responses ( $\mathrm{Li}$ and White 2003; Gauhar et al. 2009). We therefore profiled small RNA, mRNA, and protein levels throughout the ecdysone response (Fig. 2A). Specifically, small RNA-seq was conducted at $0 \mathrm{~h}$, $3 \mathrm{~h}$ (early response), and $48 \mathrm{~h}$ (late response) after treatment with ecdysone, and protein levels determined by label-free quantitative mass spectrometry at 0 and $48 \mathrm{~h}$. We have previously characterized the mRNA dynamics at 0,3 , and $48 \mathrm{~h}$ that we consider here in our analysis (Wood et al. 2011). Transcriptional profiling studies suggest that Drosophila Kc167 cells are plasmatocytes (Cherbas et al. 2011), a subclass of hemocytes involved in immune surveillance that may further differentiate into lamellocytes, podocytes, or macrophages (Stofanko et al. 2010). To examine whether ecdysone 
A
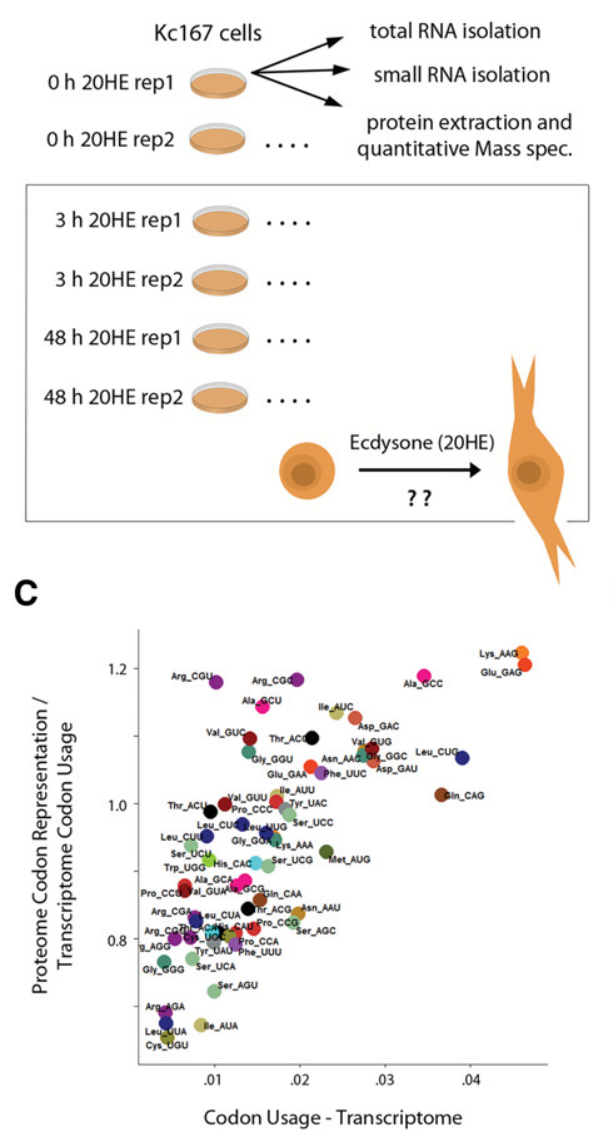

B

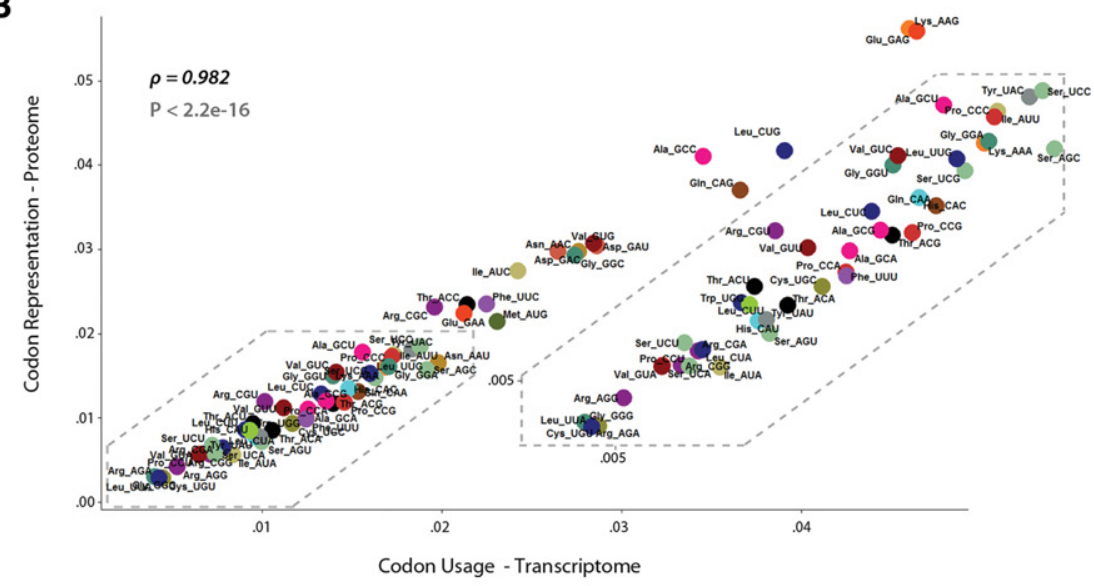

E

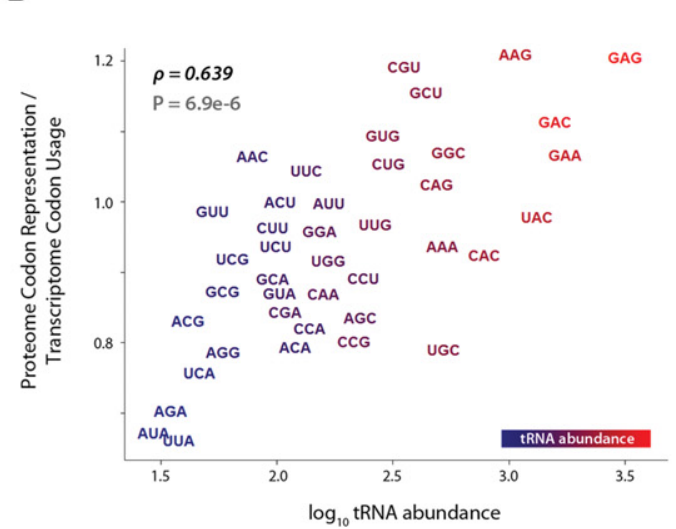

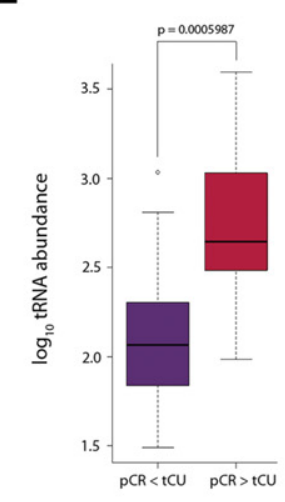

FIGURE 2. tRNA abundance scales with biased codon representation in the Kc167 cell proteome. $(A)$ Experimental design for integrated tRNA, transcript, and protein profiling in Drosophila Kc167 cells during the temporal-specific early and late ecdysone responses. Small RNA and mRNA analyses were performed on biological replicates at 0,3 , and $48 \mathrm{~h}$ after treatment with ecdysone. Label-free quantitative mass spectrometry was performed at 0 and $48 \mathrm{~h}$ on cellular lysates matched to small RNA-seq experiments. $(B)$ Correlation plot for transcriptional codon usage $(x$ axis) and codon representation in the proteome at 0 h. $\rho=0.982$; Spearman's rank correlation, $P<2.2 \times 10^{-16}$. (C) Biased codon representation in the proteome compared with transcriptomic codon usage frequencies ( $y$-axis) as a function of transcriptomic codon usage $(x$-axis). (D) Correlation plot for biased proteomic codon representation ( $y$-axis) with tRNA levels $\left(\log _{10}\right.$ tRNA abundance, as measured by small RNA-seq experiments; $x$-axis). $\rho=0.639$; Spearman's rank correlation, $P=6.9 \times 10^{-6}$. (E) Box and whisker diagram comparing the abundance for all tRNAs matching underrepresented codons ( $\mathrm{pCR}<\mathrm{tCU}$, blue) with tRNAs matching overrepresented codons ( $\mathrm{pCR}>\mathrm{tCU}$, red), comparing the proteome with the transcriptome. (pCR) Proteomic codon representation, (tCU) transcriptomic codon usage. $P=5.9 \times 10^{-4}$, Wilcoxon rank sum test.

treatment has an effect on the differentiation state of Kc167 cells, we analyzed expression markers for these relevant cell types at 0,3 , and $48 \mathrm{~h}$. Up-regulation of Croquemort, a macrophage receptor for apoptotic cells (Franc et al. 1996, 1999), Singed (Fascin), an actin-bundling protein important for macrophage migration (Zanet et al. 2012), and Schnurri, previously shown to regulate hemocyte-to-macrophage differentiation (Kelsey et al. 2012), together suggest that ecdysone induces plasmatocyte-to-macrophage differentiation in this cell line (Fig. 3A).

Label-free quantitative mass spectrometry on cellular lysates at 0 and $48 \mathrm{~h}$ identifies 276 proteins significantly increasing and 129 proteins decreasing after treatment with ecdysone (FDR $=5.2 \%$, Supplemental Fig. S4). We find that changes in protein levels agree well with the concomitant transcriptional dynamics after $48 \mathrm{~h}$, suggesting that changes in transcript levels directly influence protein abundance (Fig. 3B, $P<2.2 \times 10^{-16}$, Spearman's rank correlation). Small RNA-seq analysis reveals several miRNAs that significantly increase or decrease in response to 20-HE. The Let-7 cluster, for example, includes three highly conserved developmental miRNAs known to be up-regulated specifically during the late ecdysone response (Bashirullah et al. 2003). Accordingly, we observe significant enrichment of small RNA-seq reads at the Let-7 cluster specifically after $48 \mathrm{~h}$ (Fig. 3C). Conversely, miRNAs miR-14 and miR-34, which target the ecdysone receptor and other ecdysone responsive genes, are significantly down-regulated after $48 \mathrm{~h}$. These results suggest that our transcript, protein, and small RNA profiles accurately reflect the ecdysone response in Drosophila Kc167 cells. 
A

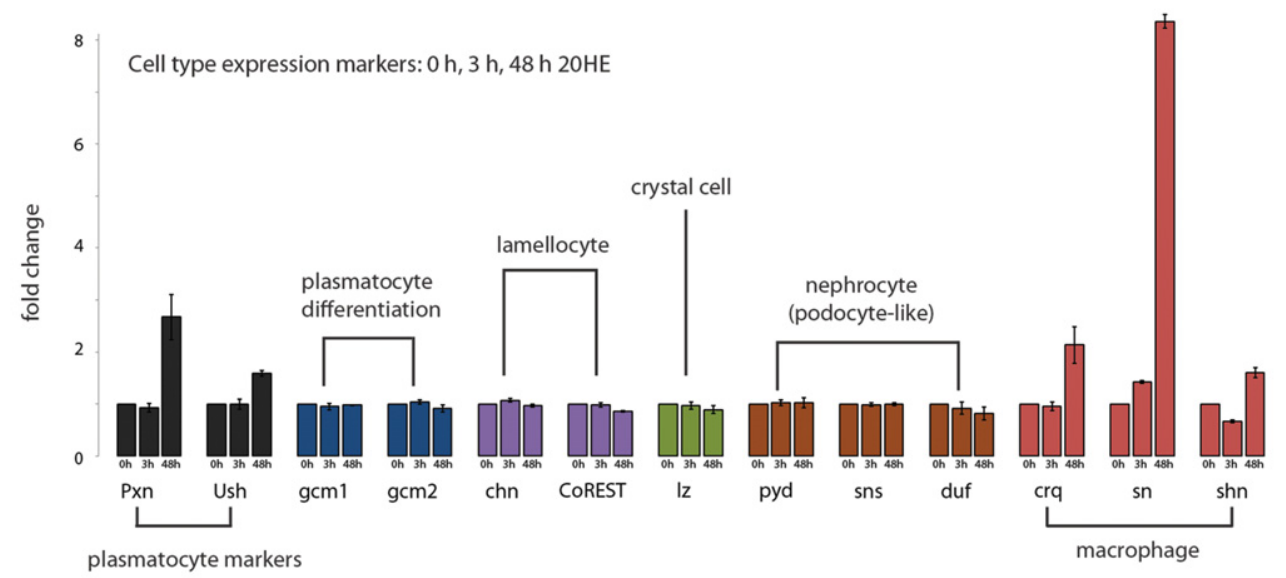

B

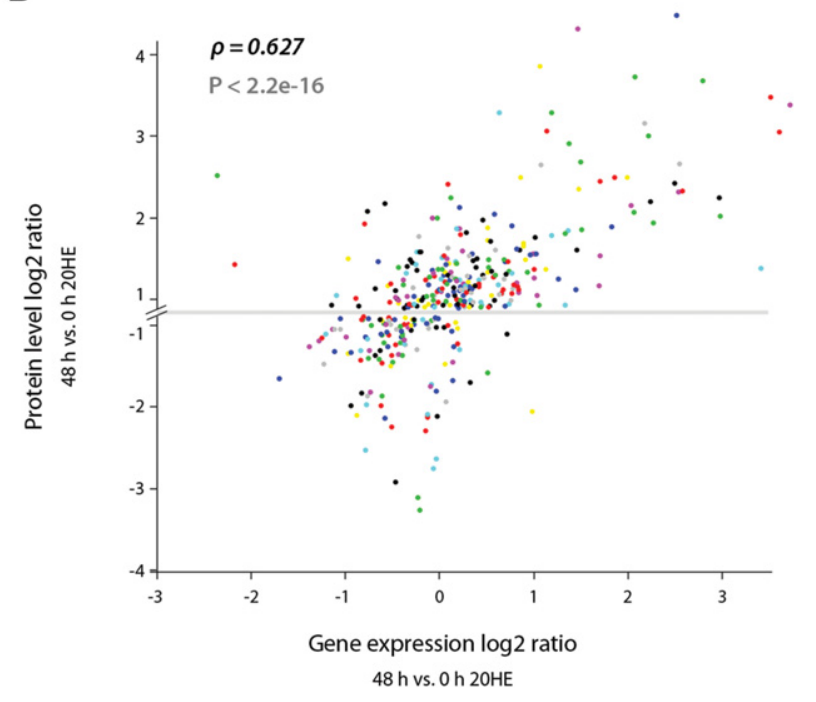

C
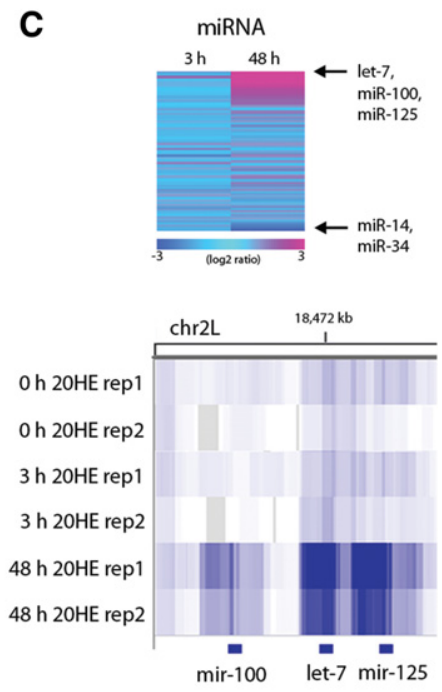

FIGURE 3. Transcriptional, proteomic, and small RNA response to ecdysone-induced cellular differentiation. (A) Expression of relevant cellular identity and differentiation pathway genes at 0,3 , and $48 \mathrm{~h}$ suggests ecdysone induces plasmatocyte-to-macrophage differentiation after $48 \mathrm{~h}$. (B). Correlation of significantly increasing and decreasing proteins identified by label-free quantitative mass spectrometry ( $\log _{2}$ ratio, $y$-axis) with changes in gene expression ( $\log _{2}$ ratio, $x$-axis) after 48 h. $\rho=0.627$; Spearman's rank correlation, $P<2.2 \times 10^{-16}$. (C) Validation of small RNA-seq experiments and response to ecdysone. Heatmap representation of miRNA expression $\left(\log _{2}\right.$ ratio) during the early and late response identifies significant changes for previously characterized ecdysone-response miRNAs (top). For example, temporal-specific expression of the Let-7 miRNA cluster during the late ecdysone response (bottom). Heatmap taken from IGV Genome Browser (Thorvaldsdottir et al. 2013).

\section{Dynamic codon usage and codon usage bias in response to ecdysone-induced differentiation}

Analysis of transcript codon usage across 0,3 , and $48 \mathrm{~h}$ ecdysone time points reveals significant changes in the frequency with which specific codons are used by the Kc167 transcriptome, most notably after $48 \mathrm{~h}$ (Fig. 4A,B). In particular, we find that codon triplets enriched in differentiation-associated genes, obtained using the Gene Ontology Consortium (GO:0030154) increase in codon usage after $48 \mathrm{~h}$, whereas codon triplets enriched in proliferation-associated genes (cell cycle, GO:0007049) decrease after 48 h (Fig. 4B, $P<$ $2.2 \times 10^{-16}$, Spearman's rank correlation). This result is consistent with the differentiation of Kc167 cells into macrophages after $48 \mathrm{~h}$, and with recent reports suggesting that differentiation- and proliferation-associated genes are enriched for distinct codons (Gingold et al. 2014).

Aggregate analysis of amino acid enrichment suggests that, compared with proliferation genes, differentiation-associated genes are also enriched for specific amino acids that increase after treatment with ecdysone (Fig. 4C,D). Since increasing or decreasing the frequency of specific amino acids is likely to have a significant influence over general codon usage, we next analyzed whether the ecdysone-induced transcriptome favored specific synonymous codons, a phenomenon called Codon Usage Bias (CUB). Instead of comparing the frequency of a given codon to all other codons, CUB takes into account the relative frequency of a codon to all other synonymous codons that encode for the same amino acid (Fig. 4E, F; Supplemental Fig. S2). This analysis reveals that, after 48 h, 


\section{Earlyecdysone response}

A

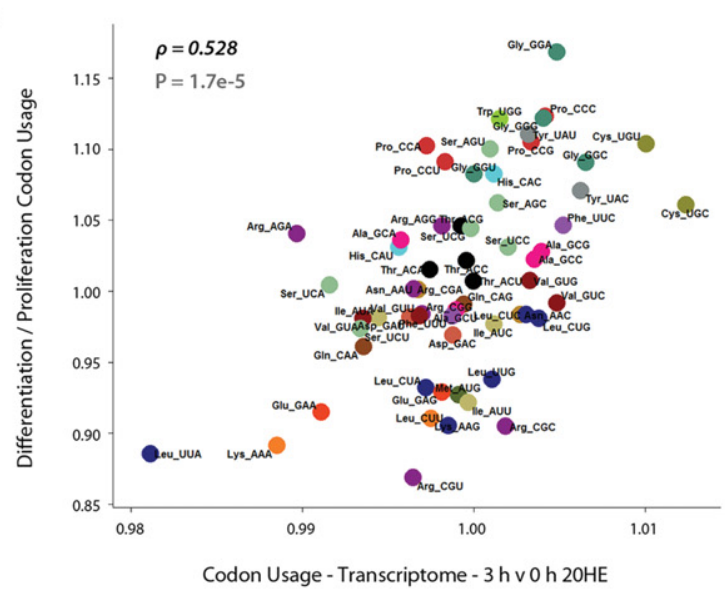

C

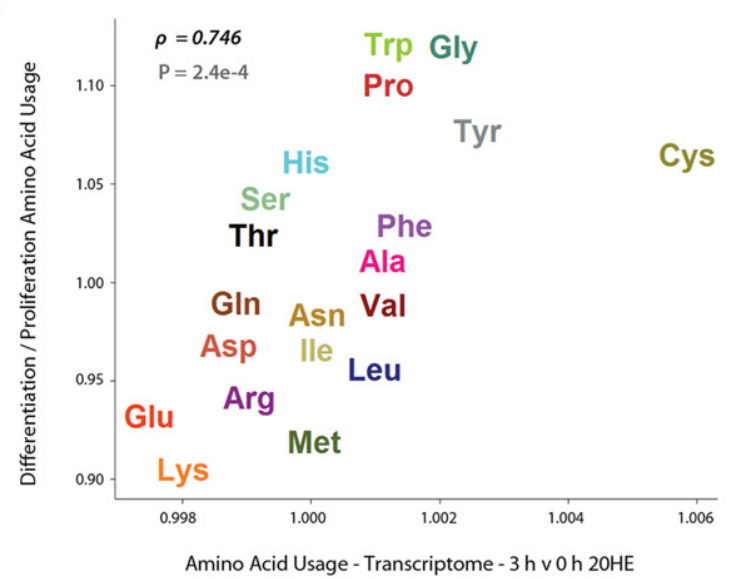

E

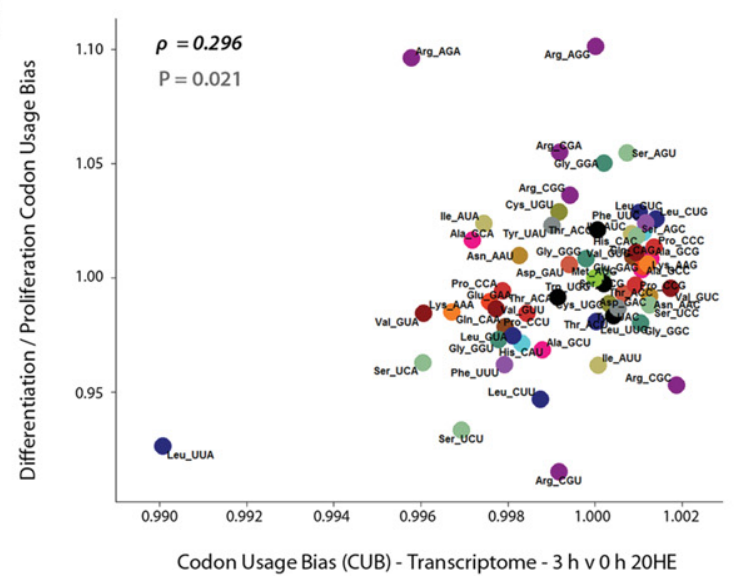

Late ecdysone response

B

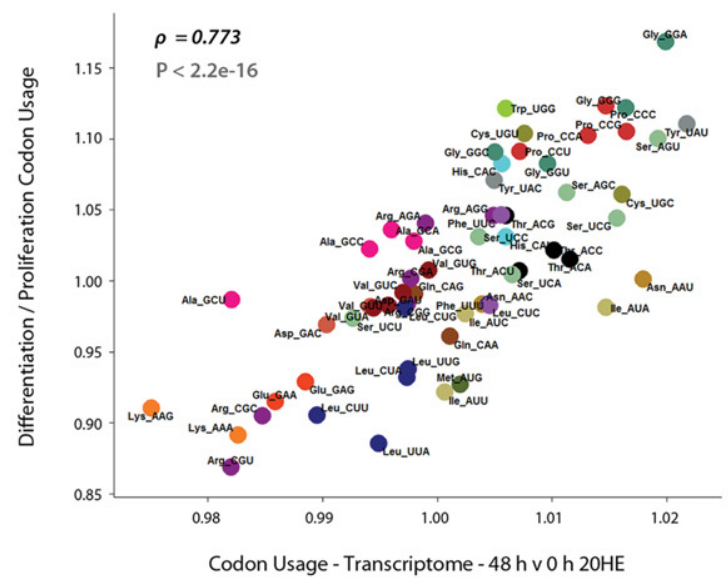

D

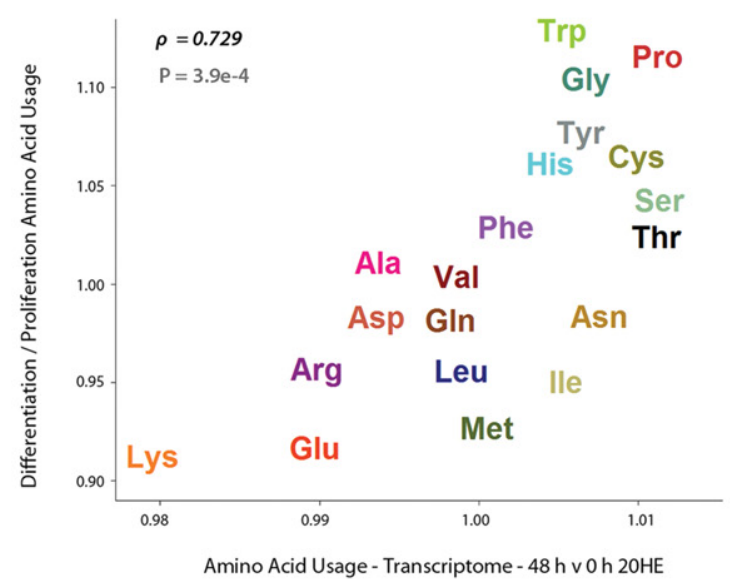

$\mathbf{F}$

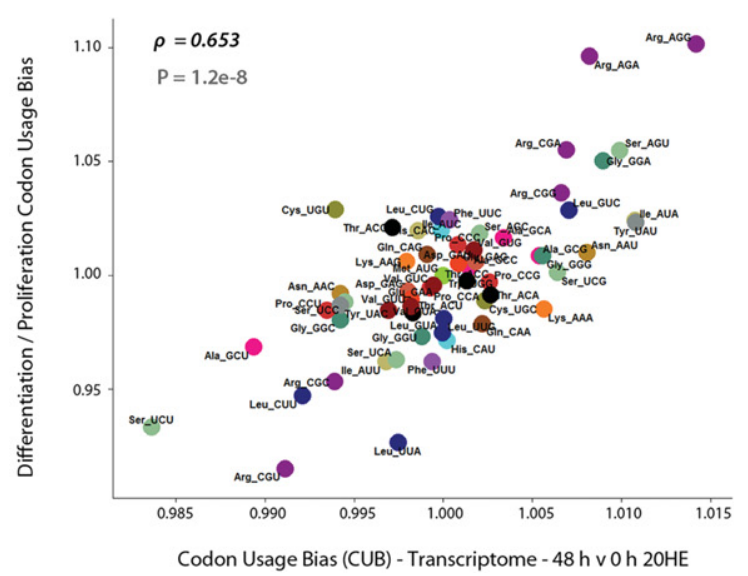

FIGURE 4. Dynamic codon usage in the Drosophila transcriptome correlates with cellular differentiation status. (A) Correlation analysis for changes in codon usage in the transcriptome after $3 \mathrm{~h}$ ecdysone treatment, with codons that are enriched or depleted in differentiation genes. $\rho=0.528$; Spearman's rank correlation, $P=1.7 \times 10^{-5}$. ( $y$-axis) Codon usage frequency for genes with differentiation gene ontology (GO) term divided by codon usage frequency for genes with proliferation (cell cycle) GO. Individual codons are color-coded by the relevant amino acid. (B) Analogous correlation plot for changes in transcriptional codon usage after 48 h. $\rho=0.773$; Spearman's rank correlation, $P<2.2 \times 10^{-16}$. (C) Correlation plot for changes in amino acid usage (aggregate of all synonymous codons) in the transcriptome after $3 \mathrm{~h}$ with amino acid usage in differentiation versus proliferation genes. $\rho=0.746$; Spearman's rank correlation, $P=2.4 \times 10^{-4}$. $(D)$ Analogous correlation plot for changes in amino acid frequency in the transcriptome after 48 h. $\rho=0.729$; Spearman's rank correlation, $P=3.9 \times 10^{-4}$. (E) Correlation analysis for differences in codon usage bias across the $3 \mathrm{~h}$ transcriptome compared with codon usage bias in differentiation versus proliferation genes. $\rho=0.296$; Spearman's rank correlation, $P=0.021$. $(F)$ Correlation analysis for differences in codon usage bias in the $48 \mathrm{~h}$ transcriptome compared with codon usage bias in differentiation versus proliferation genes. $\rho=0.653$; Spearman's rank correlation, $P=1.2 \times 10^{-8}$. 
the Kc167 transcriptome favors synonymous codons that are enriched in differentiation genes (Fig. 4F, $P=1.2 \times 10^{-8}$, Spearman's rank correlation). Together, these results provide evidence suggesting that ecdysone-induced differentiation influences both codon usage and codon usage bias, most notably in the transcriptome during the late $48 \mathrm{~h}$ time point (Fig. 4B,D,F). We therefore next asked whether these changes become evident in the proteome and to what degree tRNA levels are influenced by ecdysone.

\section{Codon representation in the late proteome mirrors the early transcriptional response}

As described for transcript codon usage analyses, we compared codon representation in the Kc167 proteome before treatment and $48 \mathrm{~h}$ after treatment with ecdysone, taking into account the abundance of each protein. Correlation analysis for changes in proteomic and transcriptomic amino acid frequencies reveals a significant relationship between the late proteomic and early transcriptional response (Fig. 5A, $P=$ $2.4 \times 10^{-4}$, Spearman's rank correlation), but not between the late proteomic and late transcriptional response (Fig. $5 B, P>0.05)$. Similarly, changes in transcript codon usage and proteomic codon representation significantly correlate between the early transcript/late proteomic response (Fig. $5 \mathrm{C}, P=1.2 \times 10^{-4}$, Spearman's rank correlation), but not between the late transcript/late proteomic response (Fig. 5D, $P$ $=0.035$ ). These results suggest that whereas early changes in transcription are reflected in the late stage proteome, changes in transcript abundance after $48 \mathrm{~h}$ may require more time to become efficiently translated and evident in the proteomic landscape. A second possibility is that tRNA abundance becomes a limiting factor in how efficiently changes in codon usage can be translated into protein. Therefore, we next asked whether ecdysone influences the relative abundance for each tRNA type, and whether a relationship may exist between tRNA dynamics and proteomic codon representation.

\section{Early and late tRNA dynamics in response to 20-hydroxyecdysone}

Analysis of tRNA levels after a $3 \mathrm{~h}$ treatment with ecdysone shows that minimal changes occur for most tRNA types, and that the relative hierarchy of tRNA abundance is maintained (Fig. 5E; Supplemental Fig. S5). However, the most significantly increasing tRNA molecule, tRNA ${ }^{\mathrm{Thr}(\mathrm{CGU})}$, increases $\sim 85 \%$ after $3 \mathrm{~h}$ of ecdysone treatment, and specifically decodes a differentiation-gene enriched codon, ACG, that increases in proteomic codon representation after $48 \mathrm{~h}$ despite a slight decrease in transcriptional codon usage (Fig. 5C). The level of tRNA ${ }^{\mathrm{Thr}(\mathrm{CGU})}$ continues to increase after $48 \mathrm{~h}$ (Fig. 5F), during which the transcript codon usage for ACG also increases compared with nontreated Kc167 cells (Fig. 5D). We speculate that by increasing the level of tRNA $^{\text {Thr(CGU) }}$, one of the least abundant tRNAs in Kc167 cells, the translational efficiency for transcripts carrying this suboptimal codon may also increase, leading to an increase in proteomic codon representation.

In general, tRNA levels fluctuate more dramatically after $48 \mathrm{~h}$, though the relative abundance for most tRNAs continues to be maintained. In addition to tRNA ${ }^{\mathrm{Th}(\mathrm{CGU})}$, tRNAs corresponding to codons Leu(CUU), Gly(GGA), Gly(GGC), and Asn(AAC) increase $>40 \%$ after $48 \mathrm{~h}$ (Fig. $5 \mathrm{~F})$, and all but Leu(CUU) are enriched in differentiation genes. Only tRNA ${ }^{\mathrm{Ile}(\mathrm{UAU})}$, which corresponds to a proliferation-gene enriched codon, Ile(AUA), decreases $>40 \%$ after $48 \mathrm{~h}$ (Fig. 5F). While these results suggest some bias in the up- and down-regulation of specific tRNAs, we find no statistically significant correlation between changes in codon usage or proteomic codon representation with changes in tRNA abundance. We speculate that ecdysone-induced changes in transcript codon usage may not require significant adjustment of tRNA levels, but that other cell types with more distinct codon repertoires may inherently require unique tRNA programs. Nevertheless, the correlation between tRNA abundance and proteomic codon representation in untreated Kc167 cells continues to hold true after $48 \mathrm{~h}$ (Supplemental Fig. S6), suggesting that tRNA levels are an important player in proteomic output.

\section{DISCUSSION}

We present an integrated analysis describing a relationship between tRNA abundance, codon usage, and protein synthesis. High abundance tRNAs correlate with codons that are overrepresented in the Drosophila proteome compared with the observed transcript codon usage. Conversely, tRNAs that are in low supply are represented in the proteome with lower frequency than observed in the transcriptome, together suggesting that tRNA abundance influences the translational output of the cell. These results are in agreement with recent studies identifying tRNA levels as an important feature in the rate of translation elongation. Inhibition of histidine biosynthesis in yeast, for example, causes a significant increase in ribosome occupancy specifically at histidine codons CAU and CAC (Lareau et al. 2014). Similarly, oxidative stress in yeast leads to increased ribosome pausing at certain aspartic acid and serine codons and a loss of ribosome occupancy at others, suggesting that this effect is governed by tRNA availability rather than amino acid synthesis (Pelechano et al. 2015). Indeed, fluctuation of tRNA levels in response to oxidative stress has been observed in Saccharomyces cerevisiae (Pang et al. 2014), and additional studies suggest that the levels of specific tRNA modifications also change dramatically in a stress-response-specific manner (Chan et al. 2010, 2012).

We speculate that, in addition to changes in tRNA abundance, reprogramming of tRNA modifications may also represent an important mechanism for regulating cell-typespecific translation patterns in Drosophila. For example, exposure of yeast to distinct toxicants leads to reprogramming 


\section{Early ecdysone response}

\section{A}

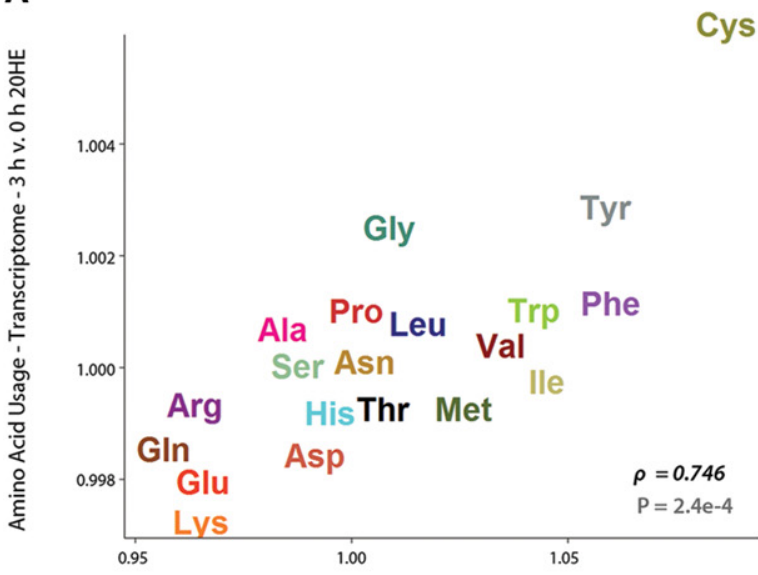

Amino Acid Representation - Proteome -48 h v. 0 h $20 \mathrm{HE}$
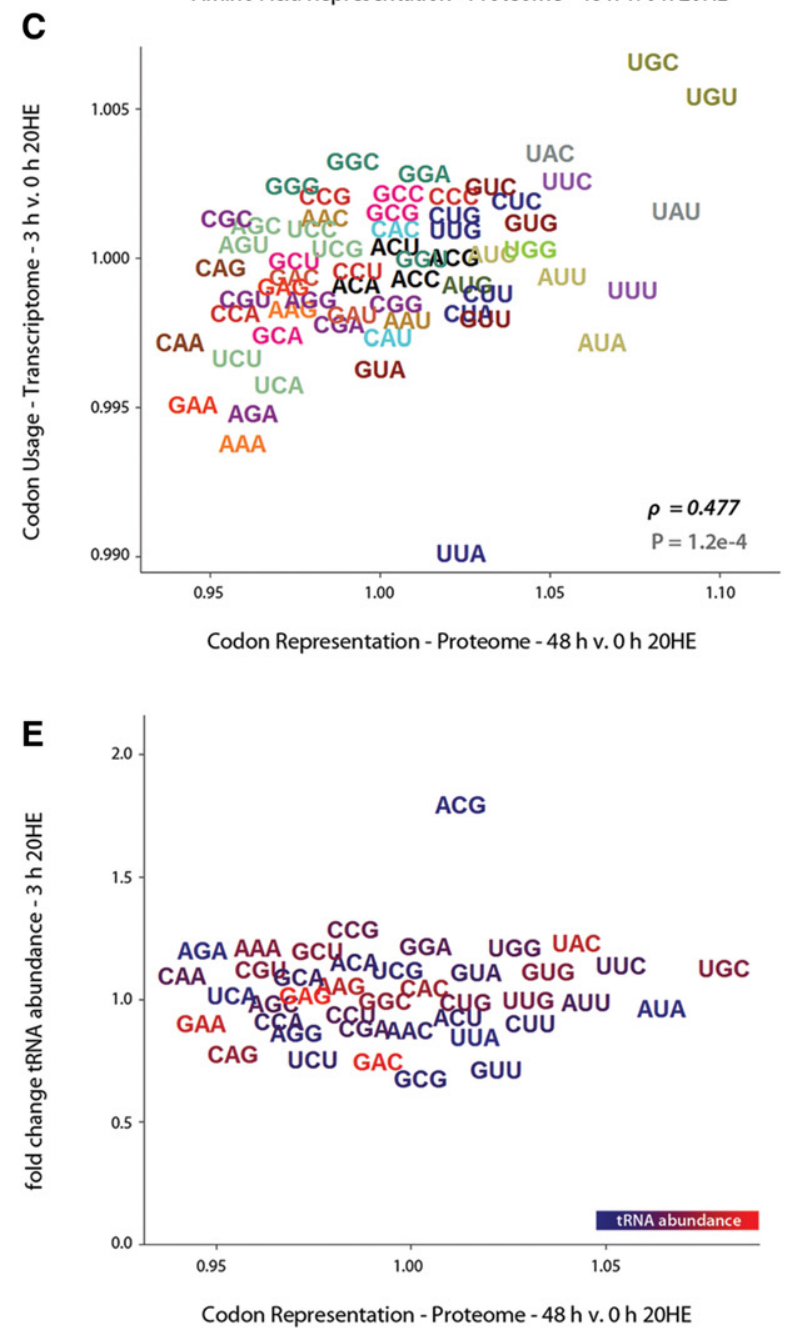

Late ecdysone response

B

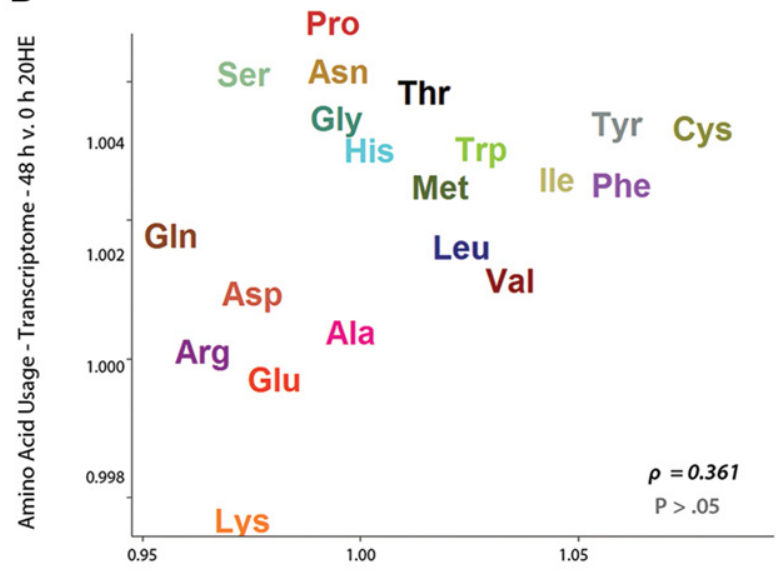

Amino Acid Representation - Proteome - 48 h v. 0 h $20 \mathrm{HE}$

D
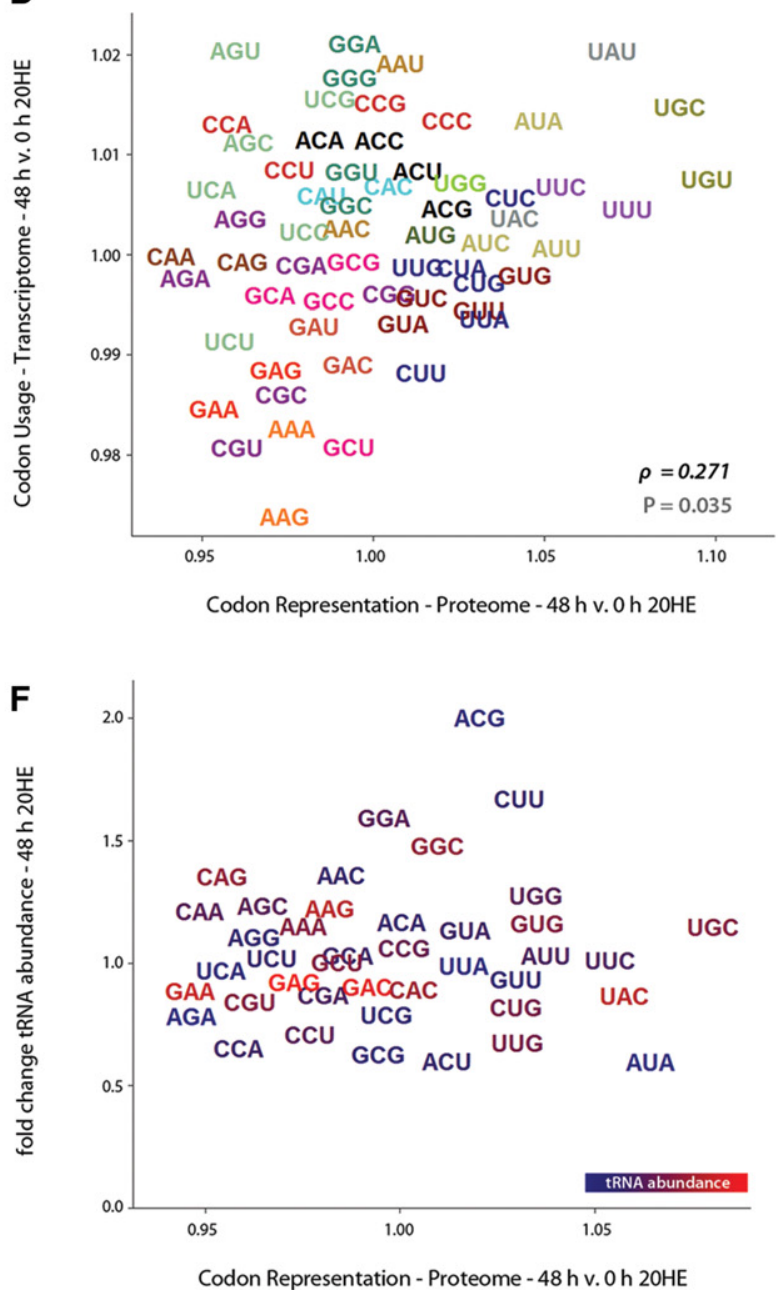

FIGURE 5. Codon representation in the late ecdysone proteome closely mirrors the early ecdysone transcriptional response. (A) Correlation analysis for changes in amino acid representation across the $48 \mathrm{~h}$ proteome with changes in amino acid frequency (aggregate usage of all synonymous codons) in the transcriptome at $3 \mathrm{~h} . \rho=0.746$; Spearman's rank correlation, $P=2.4 \times 10^{-4}$. (B) Analogous correlation plot for changes in amino acid representation after $48 \mathrm{~h}$ with changes in amino acid frequency in the transcriptome after 48 h. $\rho=0.361$; Spearman's rank correlation, $P>0.05$. $(C)$ Correlation plot for changes in codon representation in the $48 \mathrm{~h}$ proteome with transcriptional codon usage after 3 h. $\rho=0.477$; Spearman's rank correlation, $P=1.2 \times 10^{-4}$. $(D)$ Analogous correlation plot for transcriptional codon usage after $48 \mathrm{~h} . \rho=0.271$; Spearman's rank correlation, $P=$ 0.035. (E) Correlation analysis for codon representation in the proteome after $48 \mathrm{~h}$ with changes in tRNA abundance during the early, $3 \mathrm{~h}$ ecdysone response. $(F)$ Analogous correlation analysis for changes in tRNA abundance during the late, $48 \mathrm{~h}$ ecdysone response. 
of tRNA wobble modifications and altered translation patterns (Chan et al. 2015), consistent with reports suggesting that wobble base-pairing otherwise causes significant ribosome pausing (Stadler and Fire 2011). Studies probing tRNA modification in Drosophila melanogaster have identified modification of guanosine to queuosine in the wobblebinding position (Owenby et al. 1979), with varying levels of modification present during development and across other Drosophila species (Zaborske et al. 2014). Queuosine modification depends on nutrient availability and leads to increased accuracy of codon recognition, suggesting that environmental context can dramatically influence tRNA modification and consequently translation. Though a tRNA-specific adenosine deaminase, ADAT1, has been identified in D. melanogaster (Keegan et al. 2000), the effects of A to I tRNA editing in Drosophila have remained largely uncharacterized.

A recent study measuring transcript and tRNA levels during tissue development similarly identified a tight correlation between codon usage and tRNA abundance in mice (Schmitt et al. 2014). Codon representation was reported to be largely invariant during development, suggesting a remarkably stable interface between codon usage and tRNA pools in the tissues tested. By profiling the tRNAome, transcriptome, and proteome in Drosophila Kc167 cells in response to ecdysone, we however demonstrate that steroid-hormone signaling induces plasmatocyte-to-macrophage differentiation in Kc167 cells and, in so doing, shifts codon usage and codon usage bias in favor of codons preferred by differentiation genes. This effect becomes most pronounced in the transcriptome after $48 \mathrm{~h}$, reflecting the differentiation state of the cell. The dynamic, differentiation-associated codon usage we observe is consistent with a recent report describing distinct codon preferences in proliferative and differentiated cells (Gingold et al. 2014). We similarly demonstrate that, like proliferative and differentiated cell types, ecdysone-induced differentiation of Kc167 cells leads to changes in tRNA abundance in a pattern also consistent with the developmental progression of the cell. For example, tRNA ${ }^{\mathrm{Thr}(\mathrm{CGU})}$, which increases at both early and late time points, correlates with increased proteome codon representation despite minimal fluctuation in transcript codon usage, suggesting that by changing the abundance of $\mathrm{tRNA}^{\mathrm{Thr}(\mathrm{CGU})}$, translational efficiency of the cognate codon may be improved. One limitation of our analysis is that nearly one-third of all tRNA types remain missing in the current annotation database for Drosophila tRNA genes, which rely on predictive algorithms for assigning gene identity. This suggests that either current algorithms cannot accurately predict all Drosophila tRNA types or that the current reference genome sequence is missing genomic regions corresponding to tRNA genes. Despite this limitation, analysis of tRNA dynamics for codons in which annotations for all synonymous codons are available (Gln, Glu, and Lys) suggests that, after $48 \mathrm{~h}$, tRNA abundance is dynamically altered in a manner that relates to differentiation-associated codon usage bias.
Potential differences in mRNA and protein stability must also be considered as mechanisms contributing to biased codon representation. Genome-wide RNA decay profiling in yeast has revealed strong biases for optimal and nonoptimal codons in stable and unstable mRNAs, respectively (Presnyak et al. 2015). However, codon usage predicts both messenger half-life and translation elongation rate, suggesting that changes in tRNA abundance, and consequently codon optimality, may itself drive changes in mRNA stability, independent of transcription. Our analysis also cannot discriminate between changes in transcription and changes in tRNA stability, as tRNAs are heavily modified post-transcriptionally in a manner that affects both the stability and maturity of the tRNA isoacceptor molecule (Phizicky and Hopper 2010). Nevertheless, the variation in tRNA abundance after treatment with ecdysone suggests that different cell types may possess unique tRNA repertoires that are perhaps tuned toward cell-type-specific transcriptional codon usage. Because tRNA abundance appears to play a role in translational efficiency, differences in tRNA levels between cell types would likely also have a significant impact on the diversity of the proteome. Recent whole-genome sequencing studies have identified significant tRNA gene copy variation ( $\operatorname{tgCNV}$ ) between individuals (Iben and Maraia 2014). Whether tgCNV leads to differences in tRNA abundance among human populations, and consequently, whether tgCNV influences proteome diversity remains an important and unaddressed avenue for future research.

\section{MATERIALS AND METHODS}

\section{Cell culture and 20-hydroxyecdysone treatment}

Kc167 cells were grown in CCM3 serum-free insect media (HyClone $\mathrm{SH} 30065.01)$ at $25^{\circ} \mathrm{C}$. For ecdysone treatment, cells were plated to $0.5 \times 10^{6}$ cells $/ \mathrm{mL}$ and grown overnight. Cells were treated the following morning with 20-hydroxyecdysone (Sigma) at a final concentration of $5 \times 10^{-7} \mathrm{M}$ for 3 or $48 \mathrm{~h}$. Cells in both 0 h experiments were treated with ethanol as a control for ethanol-dissolved ecdysone treatment. Proteomics and small RNAs were then harvested from Kc167 cellular lysates.

\section{Small RNA-seq}

Small RNAs $<200$ nt were enriched from total RNA samples purified from Kc167 cells using the mirVana Isolation Kit (Life Technologies; cat \# AM1560), without any subsequent size selection. Small RNA library preparation and sequencing was performed by the Genomic Services Laboratory at HudsonAlpha. Small RNAsequencing reads and adaptor sequences were trimmed using the ngsShort toolkit (Chen et al. 2014). Sequencing reads were subsequently mapped to the Dm6 release of the Drosophila genome (Hoskins et al. 2015) using bowtie1.0.0 (Langmead 2010), allowing one mismatch and randomly assigning multimapping reads a single alignment to the highest scoring region. Exact read duplicates, which arise from PCR amplification were removed using samtools 
(Li et al. 2009). Sequencing replicates (2) within each biological replicate (2) were combined and tRNA read counts for each annotated tRNA gene, including $15 \mathrm{nt}$ upstream and downstream, were then extracted using bedtools (Quinlan 2014). Differential expression normalization and analysis was performed using DEGseq (Wang et al. 2010). Cumulative fold change for each codon was calculated by summing normalized read counts across each tRNA isoacceptor pool and taking the average fold change between conditions across all biological replicates. For miRNA analyses, small RNA-sequencing reads were mapped to annotated miRNAs, and differences in miRNA levels expressed as $\log _{2}$ normalized read ratios.

\section{Label-free quantitative mass spectrometry}

Whole cell lysates for each biological replicate, matched with small RNA-seq samples, were submitted for label-free quantitative mass spectrometry analysis by the Emory University Proteomics Core as recently described (Donovan et al. 2013). Briefly, protein extracts were separated by SDS-PAGE in a $10 \%$ acrylamide gel, which was subsequently cut into three molecular weight regions. Gel slices were digested overnight with trypsin and peptides extracted with $5 \%$ formic acid, 50\% ACN, and dried in a SpeedVac concentrator. Purified peptides were analyzed for each biological replicate by reverse-phase liquid chromatography coupled with tandem mass spectrometry (LC-MS/MS). A total of 405 proteins were identified as significantly changing after $48 \mathrm{~h}$, with $\log _{2}$ fold change cutoffs $(48 \mathrm{~h} / 0 \mathrm{~h})$ set at the $95 \%$ confidence interval for a Gaussian fit to the experimental data. Filtering criteria required that one or more peptides be sequenced across four samples, signal-to-noise ratio maximum measurements for each protein be greater than 10 , and coefficient of variation $<50 \%$. Using these filtering criteria, FDR for significantly changing proteins is estimated to be $5.2 \%$

\section{Codon usage and codon usage bias calculations}

Codon usage was calculated by assigning the coding sequences for all transcripts and proteins detected in transcriptomic and proteomic analyses, and counting the occurrence of every nucleotide triplet for each gene, requiring that coding sequences end with a stop codon and are a multiple of three. Transcriptomic codon usage and codon frequencies in proteomic data were normalized by the abundance of mRNAs and proteins, respectively. Specifically, codon counts for each coding sequence were multiplied by $\log _{2}$ microarray expression values (Wood et al. 2011) or by spectral counts. Codon counts were subsequently pooled for all coding sequences within specific biological replicates and conditions, and percent codon usage calculated as the frequency of using any given codon with respect to all codons. Codon usage bias was similarly calculated, and represented as the frequency of using any given codon with respect to all synonymous codons for a specific amino acid.

\section{tRNA analyses}

tRNA sequencing reads were mapped to annotated tRNA gene loci, including $15 \mathrm{nt}$ upstream and downstream from the annotated start and stop locations, and normalized $\log _{2}$ ratios between each condition were calculated using DEGseq (Wang et al. 2010). Analysis of tRNA levels was restricted by the current annotation set for tRNA species using the reference 6 release of the Drosophila melanogaster genome (Hoskins et al. 2015). To date, only 44 tRNA types, including the Selenocysteine isoacceptor, are currently annotated, whereas annotations for 19 tRNA types are currently missing, presenting a major limitation in our ability to estimate the abundance of all tRNA levels. This limitation also prevents us from calculating the relative abundance of tRNAs with respect to all synonymous codon-tRNAs decoding for all but three amino acids: Gln, Glu, and Lys (Supplemental Fig. S7). Estimations of tRNA abundance for annotated tDNAs were compared with reference genome gene copies, and with transcriptome and proteome codon usages calculated as described above, using Spearman's rank correlation analysis. tRNA abundances for codons that were observed with greater or lesser frequency in the proteome than the transcriptome were compared using the Wilcoxon-rank sum test.

\section{DATA DEPOSITION}

RNA-seq data have been deposited in GEO under accession number GSE69934.

\section{SUPPLEMENTAL MATERIAL}

Supplemental material is available for this article.

\section{ACKNOWLEDGMENTS}

We thank the Genomic Services Laboratory at the HudsonAlpha Institute for Biotechnology for their help in the preparation of RNA-seq libraries and in performing Illumina sequencing of ChIP-seq samples. We also thank the Emory University Proteomics Core for performing label-free quantitative mass spectrometry. This work was supported by US Public Health Service Award R01GM035463 from the National Institutes of Health. The content is solely the responsibility of the authors and does not represent the official views of the National Institutes of Health.

Received April 8, 2015; accepted July 16, 2015.

\section{REFERENCES}

Bashirullah A, Pasquinelli AE, Kiger AA, Perrimon N, Ruvkun G, Thummel CS. 2003. Coordinate regulation of small temporal RNAs at the onset of Drosophila metamorphosis. Dev Biol 259: 1-8.

Chan CT, Dyavaiah M, DeMott MS, Taghizadeh K, Dedon PC, Begley TJ. 2010. A quantitative systems approach reveals dynamic control of tRNA modifications during cellular stress. PLoS Genet 6: e1001247.

Chan CT, Pang YL, Deng W, Babu IR, Dyavaiah M, Begley TJ, Dedon PC. 2012. Reprogramming of tRNA modifications controls the oxidative stress response by codon-biased translation of proteins. Nat Commun 3: 937.

Chan CT, Deng W, Li F, DeMott MS, Babu IR, Begley TJ, Dedon PC. 2015. Highly predictive reprogramming of tRNA modifications is linked to selective expression of codon-biased genes. Chem Res Toxicol 28: 978-988.

Chen C, Khaleel SS, Huang H, Wu CH. 2014. Software for pre-processing Illumina next-generation sequencing short read sequences. Source Code Biol Med 9: 8.

Cherbas L, Willingham A, Zhang D, Yang L, Zou Y, Eads BD, Carlson JW, Landolin JM, Kapranov P, Dumais J, et al. 2011. The 
transcriptional diversity of 25 Drosophila cell lines. Genome Res 21: 301-314.

Donovan LE, Dammer EB, Duong DM, Hanfelt JJ, Levey AI, Seyfried NT, Lah JJ. 2013. Exploring the potential of the platelet membrane proteome as a source of peripheral biomarkers for Alzheimer's disease. Alzheimers Res Ther 5: 32.

Duret L. 2002. Evolution of synonymous codon usage in metazoans. Curr Opin Genet Dev 12: 640-649.

Franc NC, Dimarcq JL, Lagueux M, Hoffmann J, Ezekowitz RA. 1996. Croquemort, a novel Drosophila hemocyte/macrophage receptor that recognizes apoptotic cells. Immunity 4: 431-443.

Franc NC, Heitzler P, Ezekowitz RA, White K. 1999. Requirement for croquemort in phagocytosis of apoptotic cells in Drosophila. Science 284: 1991-1994.

Gauhar Z, Sun LV, Hua S, Mason CE, Fuchs F, Li TR, Boutros M, White KP. 2009. Genomic mapping of binding regions for the Ecdysone receptor protein complex. Genome Res 19: 1006-1013.

Gingold H, Tehler D, Christoffersen NR, Nielsen MM, Asmar F, Kooistra SM, Christophersen NS, Christensen LL, Borre M, Sorensen KD, et al. 2014. A dual program for translation regulation in cellular proliferation and differentiation. Cell 158: 1281-1292.

Goetz RM, Fuglsang A. 2005. Correlation of codon bias measures with mRNA levels: analysis of transcriptome data from Escherichia coli. Biochem Biophys Res Commun 327: 4-7.

Gustafsson C, Govindarajan S, Minshull J. 2004. Codon bias and heterologous protein expression. Trends Biotechnol 22: 346-353.

Hoskins RA, Carlson JW, Wan KH, Park S, Mendez I, Galle SE, Booth BW, Pfeiffer BD, George RA, Svirskas R, et al. 2015. The Release 6 reference sequence of the Drosophila melanogaster genome. Genome Res 25: 445-458.

Iben JR, Maraia RJ. 2014. tRNA gene copy number variation in humans. Gene 536: 376-384.

Ingolia NT, Lareau LF, Weissman JS. 2011. Ribosome profiling of mouse embryonic stem cells reveals the complexity and dynamics of mammalian proteomes. Cell 147: 789-802.

Kanaya S, Yamada Y, Kudo Y, Ikemura T. 1999. Studies of codon usage and tRNA genes of 18 unicellular organisms and quantification of Bacillus subtilis tRNAs: gene expression level and species-specific diversity of codon usage based on multivariate analysis. Gene 238: $143-155$.

Keegan LP, Gerber AP, Brindle J, Leemans R, Gallo A, Keller W, O'Connell MA. 2000. The properties of a tRNA-specific adenosine deaminase from Drosophila melanogaster support an evolutionary link between pre-mRNA editing and tRNA modification. Mol Cell Biol 20: 825-833.

Kelsey EM, Luo X, Bruckner K, Jasper H. 2012. Schnurri regulates hemocyte function to promote tissue recovery after DNA damage. $J$ Cell Sci 125: 1393-1400.

Langmead B. 2010. Aligning short sequencing reads with Bowtie. Curr Protoc Bioinformatics 32: 11.7.1-11.7.14.

Lareau LF, Hite DH, Hogan GJ, Brown PO. 2014. Distinct stages of the translation elongation cycle revealed by sequencing ribosome-protected mRNA fragments. Elife 3: e01257.

Li TR, White KP. 2003. Tissue-specific gene expression and ecdysoneregulated genomic networks in Drosophila. Dev Cell 5: 59-72.
Li H, Handsaker B, Wysoker A, Fennell T, Ruan J, Homer N, Marth G, Abecasis G, Durbin R; 1000 Genome Project Data Processing Subgroup. 2009. The Sequence Alignment/Map format and SAMtools. Bioinformatics 25: 2078-2079.

Novoa EM, Ribas de Pouplana L. 2012. Speeding with control: codon usage, tRNAs, and ribosomes. Trends Genet 28: 574-581.

Owenby RK, Stulberg MP, Jacobson KB. 1979. Alteration of the Q family of transfer RNAs in adult Drosophila melanogaster as a function of age, nutrition, and genotype. Mech Ageing Dev 11: 91-103.

Pang YL, Abo R, Levine SS, Dedon PC. 2014. Diverse cell stresses induce unique patterns of tRNA up- and down-regulation: tRNA-seq for quantifying changes in tRNA copy number. Nucleic Acids Res 42: e170.

Pelechano V, Wei W, Steinmetz LM. 2015. Widespread co-translational RNA decay reveals ribosome dynamics. Cell 161: 1400-1412.

Percudani R, Pavesi A, Ottonello S. 1997. Transfer RNA gene redundancy and translational selection in Saccharomyces cerevisiae. J Mol Biol 268: $322-330$.

Phizicky EM, Hopper AK. 2010. tRNA biology charges to the front. Genes Dev 24: 1832-1860.

Presnyak V, Alhusaini N, Chen YH, Martin S, Morris N, Kline N, Olson S, Weinberg D, Baker KE, Graveley BR, et al. 2015. Codon optimality is a major determinant of mRNA stability. Cell 160: $1111-1124$

Qian W, Yang JR, Pearson NM, Maclean C, Zhang J. 2012. Balanced codon usage optimizes eukaryotic translational efficiency. PLoS Genet 8: e1002603.

Quinlan AR. 2014. BEDTools: the Swiss-army tool for genome feature analysis. Curr Protoc Bioinformatics 47: 11.12.11-11.12.34.

Schmitt BM, Rudolph KL, Karagianni P, Fonseca NA, White RJ, Talianidis I, Odom DT, Marioni JC, Kutter C. 2014. High-resolution mapping of transcriptional dynamics across tissue development reveals a stable mRNA-tRNA interface. Genome Res 24: 1797-1807.

Stadler M, Fire A. 2011. Wobble base-pairing slows in vivo translation elongation in metazoans. RNA 17: 2063-2073.

Stofanko M, Kwon SY, Badenhorst P. 2010. Lineage tracing of lamellocytes demonstrates Drosophila macrophage plasticity. PLoS One 5: e14051.

Thorvaldsdottir H, Robinson JT, Mesirov JP. 2013. Integrative Genomics Viewer (IGV): high-performance genomics data visualization and exploration. Brief Bioinform 14: 178-192.

Wang L, Feng Z, Wang X, Zhang X. 2010. DEGseq: an R package for identifying differentially expressed genes from RNA-seq data. Bioinformatics 26: 136-138.

Wood AM, Van Bortle K, Ramos E, Takenaka N, Rohrbaugh M, Jones BC, Jones KC, Corces VG. 2011. Regulation of chromatin organization and inducible gene expression by a Drosophila insulator. Mol Cell 44: 29-38.

Zaborske JM, DuMont VL, Wallace EW, Pan T, Aquadro CF, Drummond DA. 2014. A nutrient-driven tRNA modification alters translational fidelity and genome-wide protein coding across an animal genus. PLoS Biol 12: e1002015.

Zanet J, Jayo A, Plaza S, Millard T, Parsons M, Stramer B. 2012. Fascin promotes filopodia formation independent of its role in actin bundling. J Cell Biol 197: 477-486. 

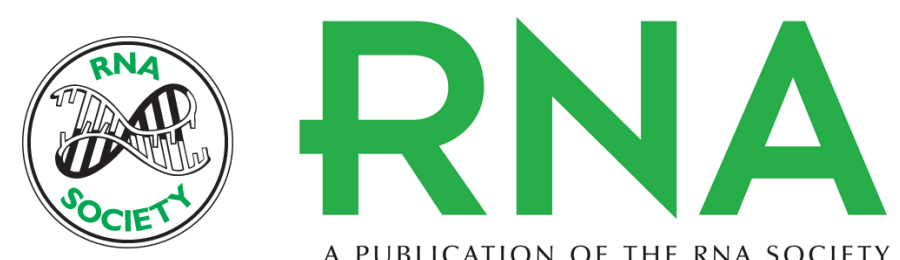

A PUBLICATION OF THE RNA SOCIETY

\section{Integrated tRNA, transcript, and protein profiles in response to steroid hormone signaling}

Kevin Van Bortle, Michael H. Nichols, Edward Ramos, et al.

RNA 2015 21: 1807-1817 originally published online August 19, 2015

Access the most recent version at doi:10.1261/rna.052126.115

\section{Supplemental http://rnajournal.cshlp.org/content/suppl/2015/08/07/rna.052126.115.DC1 Material}

References This article cites 41 articles, 10 of which can be accessed free at: http://rnajournal.cshlp.org/content/21/10/1807.full.html\#ref-list-1

Creative This article is distributed exclusively by the RNA Society for the first 12 months after the Commons License full-issue publication date (see http://rnajournal.cshlp.org/site/misc/terms.xhtml). After 12 months, it is available under a Creative Commons License (Attribution-NonCommercial 4.0 International), as described at http://creativecommons.org/licenses/by-nc/4.0/.

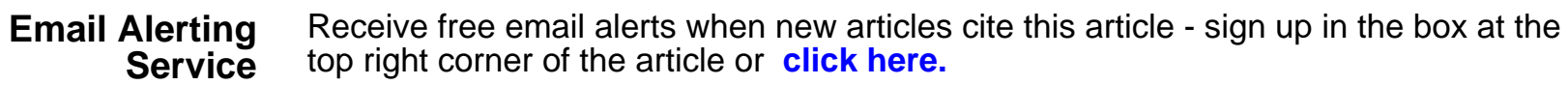

